Tohoku J. Exp. Med., 1995, 176, 75-81

\title{
A Method of Short-Term Cryostorage and Selection of Viable Sperm for Use in the Various Assisted Reproductive Techniques ${ }^{1}$
}

\author{
Panayiotis M. Zavos*† П, Juan R. Correa*†, Nikolas \\ Sofikitis $\ddagger$ George D. Kofinas§ and Panayota N. \\ ZarmakouPIS// \\ *Andrology Institute of Lexington, $\uparrow$ Department of Animal \\ Sciences, University of Kentucky, Lexington, KY, USA; \\ $\ddagger$ Department of Urology, Tottori University School of \\ Medicine, Yonago 683, Japan; §The Brooklyn Hospital \\ Center, Brooklyn, NY, USA; /Department of $O B / G Y N$, \\ University of South Florida, Tampa, FL, USA; and \\ ๆ Fertility Institute of Athens, Athens, Greece
}

Zavos, P.M., Correa, J.R., Sofikitis, N., Kofinas, G.D. and Zarmakoupis, P.N. A Method of Short-Term Cryostorage and Selection of Viable Sperm for Use in the Various Assisted Reproductive Techniques. Tohoku J. Exp. Med., 1995, 176 (2), 75-81 — The objective of this study was to determine if spermatozoa, following short-term cryostorage at $5^{\circ} \mathrm{C}$ in Test-Yolk buffer (TYB; ZBL, Inc., Lexington, KY, USA), could be recovered and improved via the SpermPrep ${ }^{\mathrm{TM}}$ filtration method and to assess the possibly enhanced fertilizing capacity of the selected spermatozoa. Semen specimens from 20 men were collected, evaluated, diluted 1:1 (v/v) with TYB, divided into aliquots and cooled to $5^{\circ} \mathrm{C}$ for 24 and $48 \mathrm{hr}$. Semen samples were assessed for volume, sperm count, percentage and grade of motility, percentage of morphologically normal spermatozoa and outcome of the sperm penetration assay (SPA). After storage, aliquots were rewarmed at $37^{\circ} \mathrm{C}$, centrifuged, and the pellet was resuspended in $1.0 \mathrm{ml}$ of SpermPrep ${ }^{\mathrm{TM}}$ media (ZBL, Inc.). Following $15 \mathrm{~min}$ of incubation, the rewarmed spermatozoa were filtered via the SpermPrep ${ }^{\mathrm{TM}}$ I filtration column (ZBL, Inc.) and assessed accordingly. The results obtained in this study indicate that the short-term cryostorage procedure yielded spermatozoa of adequate qualitative characteristics when compared to the fresh spermatozoa. Furthermore, filtration of rewarmed specimens yielded spermatozoa of significantly higher qualitative characteristics and superior fertilizing capacity following a short-term cryostorage period in TYB when compared to fresh and rewarmed spermatozoa $(p<0.05)$. This method of short-term cryostorage in TYB and selection of superior spermatozoa via the SpermPrep ${ }^{\mathrm{TM}}$ filtration method could further enhance the fertilizing ability of

Received November 18, 1994; revision accepted for publication March 7, 1995.

Address for correspondence: Professor Panayiotis M. Zavos, Ed.S., Ph.D., 607 W.P. Garrigus Building, University of Kentucky, Lexington, Kentucky 40546, USA.

${ }^{1}$ Presented in part at the 7th International Congress of Andrology; Tokyo, Japan, May 2-6, 1993. 
patients who produce spermatozoa characterized by deficient capacitation, acrosome reaction and subsequent fertilization.—-SpermPrep ${ }^{\mathrm{TM}}$; cryopreserved spermatozoa; semen filtration; sperm selection

The use of preserved human semen for artificial donor insemination (ADI) is steadily increasing, although the cryopreservation techniques presently employed reduce the fertilization potential of human spermatozoa. The use of preserved spermatozoa provides the advantage of arranging patients and performance of assisted reproductive techniques (ART; such as IUI, IVF, GIFT, etc.) whenever convenient. Injury to spermatozoa is usually attributed to ice or crystal formation during freezing instead of the temperature per se (Zavos 1990). A newlyestablished technique has been introduced to optimize fertility rates and to decrease difficulty in aligning patients. This technique consists in storing fresh human spermatozoa diluted with Test-Yolk buffer (TYB) at $5^{\circ} \mathrm{C}$ for approximately 24 to $48 \mathrm{hr}$ and up to $96 \mathrm{hr}$ (Kofinas and Zavos 1992a). It has been shown that IVF rates and sperm penetration into zona-free hamster oocytes (ZFHO) are significantly enhanced when spermatozoa are diluted with TYB (Balamos et al. 1983; Katayama et al. 1989). Furthermore, an additional increase in fertility rates could be possible by selection of spermatozoa on the basis of their progressive motility and morphological characteristics (McClure et al. 1989; Free et al. 1991).

The SpermPrep ${ }^{\mathrm{TM}}$ filtration method encompasses attractive features such as fast recovery of all or most motile and morphologically normal spermatozoa in a seminal specimen, removal of dead spermatozoa and debris, and process large volumes of semen (Zavos and Centola 1990). It is well established that filtration of spermatozoa via the SpermPrep ${ }^{\mathrm{TM}}$ filtration method increases sperm quality in a manner that translates into improved sperm fertilizing ability. Higher pregnancy rates have been obtained when using spermatozoa recovered via the SpermPrep ${ }^{\mathrm{TM}}$ filtration method (Zavos and Centola 1992). Similar observations were noted with in vitro fertilizing potential studies with SpermPrep $^{\mathrm{TM}}$ and swim-up recovered spermatozoa and their ability to penetrate ZFHO (Rogers et al. 1991).

The objective of this study was to determine if spermatozoa, following short-term cryostorage at $5^{\circ} \mathrm{C}$ in TYB could be recovered and improved via the SpermPrep ${ }^{\mathrm{TM}}$ filtration method and to assess the possibly enhanced fertilizing potential of the selected spermatozoa.

\section{Materials and Methods}

\section{Semen collection and evaluation}

Semen samples were collected from 20 men who were referred to our andrological laboratory (Andrology Institute of Lexington, Lexington, KY, USA) for male infertility workup. Ejaculates were collected with exactly 4 days of abstinence each time. All patients collected their own ejaculates using the Male 
Factor Pak ${ }^{\mathrm{TM}}$ collection device (MFP; ZBL, Inc.) at intercourse (Zavos 1985; Zavos and Goodpasture 1989). After semen samples were produced and completely liquified (within 15 to $30 \mathrm{~min}$ ), each specimen was evaluated according to standard procedures recommended by the World Health Organization (WHO 1987) using a phase-contrast microscope. Semen parameters assessed included volume $(\mathrm{ml})$, sperm count $\left(\times 10^{6}\right)$, percentage of sperm motility, grade of sperm motility ( 0 to 4 ), sperm morphologic features (percentage of morphologically normal spermatozoa) and outcome of sperm penetration assay (SPA) by using ZFHO. All seminal parameters were evaluated under blind conditions by the same technician.

\section{Semen preparation}

Aliquots containing $100.0 \times 10^{6}$ spermatozoa were diluted $1: 1(\mathrm{v} / \mathrm{v})$ with TYB (ZBL, Inc.) and gradually cooled to $5^{\circ} \mathrm{C}$ for 24 and $48 \mathrm{hr}$ in a manner similar to a previously described method (Zavos et al. 1980; Kofinas and Zavos 1992a). After storage, the specimens were gently agitated and rewarmed $\left(37^{\circ} \mathrm{C}\right)$ for $15 \mathrm{~min}$, centrifuged, and the sperm pellet was resuspended in $1.0 \mathrm{ml}\left(37^{\circ} \mathrm{C}\right)$ of SpermPrep ${ }^{\mathrm{TM}}$ media (ZBL, Inc.). Following 15 min of incubation, the rewarmed spermatozoa were filtered via the SpermPrep ${ }^{\text {TM }}$ I method. The SpermPrep ${ }^{\text {TM }}$ I filtration column was used according to the manufacturer's specifications and instructions (ZBL, Inc.). It should be emphasized that proper standard laboratory techniques were employed in our laboratory during the whole filtration process. Those techniques included complete sterility and maintenance of all semen diluents, the SpermPrep ${ }^{\mathrm{TM}}$ I filter, and all other materials within a temperature range of 30 to $35^{\circ} \mathrm{C}$. Filtration was begun by placing the $1.0 \mathrm{ml}$ volume of the properly resuspended spermatozoa in the filter. The $1.0 \mathrm{ml}$ aliquot contained $100.0 \times 10^{6}$ washed and well-mixed spermatozoa. At the end of filtration (10 min) the filtrate was centrifuged, resuspended in $1.0 \mathrm{ml}$ of SpermPrep ${ }^{\mathrm{TM}}$ media and assessed as previously described.

\section{Sperm penetration assay (SPA)}

The methodology used for the SPA, including semen preparation, egg recruitment and processing, insemination and evaluation, was similar to the techniques described by Rogers (1985). The results of the SPA were reported as percentage of sperm penetration into ZFHO. Data for the various treatments applied were compared to each other using one-way ANOVA followed by the student's $t$-test.

\section{RESULTS}

The mean seminal characteristics of specimens after cryopreservation, rewarming and filtration are shown in Table 1. The sperm motility of the $24 \mathrm{hr}$ cryostored sample decreased by $8.9 \%$, the grade of motility by 0.1 points (scale 0 to 4 ), the proportion of morphologically normal spermatozoa by $8.3 \%$, and the 
TABLE 1. Characteristics of cryostored, rewarmed and filtered spermatozoa following short-term cryostorage $\left(5^{\circ} \mathrm{C}\right)$ in $T Y B$ (Means \pm s.D.)

\begin{tabular}{lcccccc}
\hline & \multicolumn{6}{c}{ Semen parameters assessed $(n=20)$} \\
\cline { 2 - 7 } \multicolumn{1}{c}{$\begin{array}{c}\text { Experimental } \\
\text { conditions }\end{array}$} & $\begin{array}{c}\text { Count } \\
\left(\times 10^{6}\right)\end{array}$ & $\begin{array}{c}\text { Motility } \\
(\%)\end{array}$ & $\begin{array}{c}\text { Grade } \\
(0 \text { to } 4)\end{array}$ & $\begin{array}{c}\text { Normal } \\
\text { Morphology } \\
(\%)\end{array}$ & $\begin{array}{c}\text { SPA } \\
(\%)\end{array}$ & $\begin{array}{c}\mathrm{TFSF}^{1} \\
\left(\times 10^{6}\right)\end{array}$ \\
\hline Fresh (control) & $100.0 \pm 0.0$ & $58.3 \pm 3.7$ & $3.3 \pm 0.2$ & $59.6 \pm 4.0$ & $41.2 \pm 3.5$ & $34.7 \pm 3.9$ \\
Rewarmed $24 \mathrm{hr}$ & $100.0 \pm 0.0$ & $49.4 \pm 4.1$ & $3.2 \pm 0.3$ & $57.3 \pm 5.1$ & $35.1 \pm 4.0$ & $28.3 \pm 3.8$ \\
Filtered $24 \mathrm{hr}$ & $41.8 \pm 4.5$ & $69.5 \pm 3.3^{*}$ & $3.7 \pm 0.2^{*}$ & $86.5 \pm 5.3^{*}$ & $55.7 \pm 4.2^{*}$ & $25.1 \pm 3.3$ \\
Rewarmed 48 hr & $100.0 \pm 0.0$ & $45.1 \pm 5.0$ & $3.1 \pm 0.3$ & $56.6 \pm 5.7$ & $33.4 \pm 4.6$ & $25.5 \pm 4.0$ \\
Filtered 48 hr & $37.6 \pm 5.2$ & $65.2 \pm 4.5^{*}$ & $3.6 \pm 0.2^{*}$ & $85.7 \pm 5.5^{*}$ & $52.8 \pm 4.3^{*}$ & $21.0 \pm 3.5$ \\
\hline
\end{tabular}

${ }^{1}$ TFSF $=$ Count $\left(\times 10^{6}\right) \times$ motility $(\%) \times$ normal morphology $(\%)$.

*Significant improvements realized for post-filtered spermatozoa between their corresponding rewarmed, pre-filtered counterparts $(p<0.05)$.

outcome of the SPA by of $6.1 \%$. The total functional sperm fraction (TFSF), which represents the total number of motile, morphologically normal spermatozoa (Zavos et al. 1984) was reduced by $6.4 \times 10^{6}$ spermatozoa. The qualitative characteristics continued to decrease slightly when spermatozoa were cryostored for 48 $\mathrm{hr}$. In all instances, when spermatozoa were rewarmed, filtered via the SpermPrep ${ }^{\mathrm{TM}} \mathrm{I}$ and reconstituted in SpermPrep ${ }^{\mathrm{TM}}$ media, noticeable increases in all qualitative characteristics were evident when compared to the rewarmed or fresh samples $(p<0.05)$. Sperm motility after $24 \mathrm{hr}$ of cryostorage, due to the SpermPrep ${ }^{\mathrm{TM}}$ I filtration, improved by $20 \%$ when compared to the rewarmed samples. The grade of motility improved by 0.5 points, the proportion of morphologically normal spermatozoa increased by approximately $30 \%$, and the outcome of the SPA increased by $21 \%$. Similar results were obtained for spermatozoa rewarmed and filtered after $48 \mathrm{hr}$ of cryostorage.

\section{Discussion}

Spermatozoa can now be stored in liquid nitrogen at subzero temperatures (as low as $-196^{\circ} \mathrm{C}$ ) and survive with relatively high fertility after thawing. However, many of the spermatozoa are killed or rendered immotile by the freezing and thawing process. The majority of the results have indicated that approximately 30 to $70 \%$ of spermatozoa that were motile prior to freezing regained their motility after thawing (Beck and Silverstein 1975). Hence, for optimum fertility and to overcome the shortcomings of frozen-thawed human semen, larger numbers of spermatozoa are used for frozen than for fresh semen at the time of AI. Spermatozoa cryostored at $5^{\circ} \mathrm{C}$ in TYB (non-frozen) can survive better for spermatozoa frozen in liquid nitrogen at subzero temperatures (Zavos et al. 1980). It has also been documented that the treatment of human spermatozoa with TYB can 
enhance their ability to penetrate ZFHO (Balamos et al. 1983). Preincubation of spermatozoa in TYB have been shown to increase the percentage of human oocytes fertilized in IVF procedures (Katayama et al. 1989). It seems that after incubation in TYB, more spermatozoa undergo capacitation and the spontaneous acrosome reaction (Biefeld et al. 1989). Subsequently, the increased percentage of acrosome-reacted spermatozoa that is seen after TYB incubation, may indicate that a larger percentage of spermatozoa acquired the ability to penetrate the ovum and account for the higher rates of fertilization observed.

With the advent of IVF and other forms of ART, it is considered important to use the best spermatozoa available from a semen sample. Recent evidence with fresh spermatozoa suggests that spermatozoa separated via various methods have a greater fertilizing capacity than spermatozoa in the whole ejaculate (Tanphaichitr et al. 1988; Katayama et al. 1989). It has been shown that filtration via the SpermPrep ${ }^{\mathrm{TM}}$ method yields high levels of morphologically normal motile spermatozoa and with possible high fertilizing potential (Sofikitis et al. 1992a, b; Zavos et al. 1992). In a recent study (Zavos and Centola 1992), SpermPrep ${ }^{\mathrm{TM}}$ prepared spermatozoa yielded higher pregnancies than convential methods such as double sperm wash $(76.1 \%$ vs $52.1 \%$, respectively). Similar observations were noted with in vitro fertilizing potential studies with SpermPrep $^{\mathrm{TM}}$ and swim-up recovered human spermatozoa and their ability to penetrate ZFHO (Rogers et al. 1991).

The results obtained in this study indicate that the cryostorage of spermatozoa yielded spermatozoa of adequate qualitative characteristics when compared to the fresh spermatozoa. Although the cryostorage process had some deleterious effects on the spermatozoa, these effects were not severe enough to destroy the spermatozoa and prevent them from achieving fertilization. Furthermore, filtration of rewarmed specimens yielded spermatozoa of significantly higher qualitative characteristics and superior fertilizing potential, following a short-term cryostorage period in TYB, when compared to both the fresh and rewarmed spermatozoa. In a similar study, filtration of cryopreserved spermatozoa yielded spermatozoa with better survival following short-term incubation at $37^{\circ} \mathrm{C}$ (Kofinas and Zavos 1992b). Spermatozoa cryostored and recovered via the SpermPrep ${ }^{\mathrm{TM}}$ filtration method can be used for multiple inseminations in an aggressive IUI program using husband's semen not requiring semen cryopreservation at subzero temperatures $\left(-196^{\circ} \mathrm{C}\right)$. This method of short-term sperm cryostorage in TYB and selection via the SpermPrep ${ }^{\mathrm{TM}} \mathrm{I}$ filter could further enhance the fertilizing ability of patients who produce spermatozoa characterized by deficient capacitation, acrosome reaction and subsequent fertilization.

\section{References}

1) Balamos, J.R., Overstreet, J.W. \& Katz, D.F. (1983) Human sperm penetration of zona-free hamster eggs after storage of the semen for 48 hours at $2^{\circ} \mathrm{C}$ to $5^{\circ} \mathrm{C}$. Fertil. 
Steril., 39, 536-541.

2) Beck, W.W. \& Silverstein, I. (1975) Variable motility recovery of spermatozoa following freeze preservation. Fertil. Steril., 26, 863-869.

3) Biefeld, P., Jeyendran, R.S. \& Zaneveld, L.J.D. (1989) Effect of TEST-yolk on the human sperm acrosome reaction. J. Androl., 20, 40P. (abstract)

4) Free, D., Stutts, L., Merryman, D., Stringfellow, E., Horseman, V. \& Honea, K. (1991) A comparison of donor semen processing techniques for use in intrauterine inseminations and their corresponding pregnancy rates. Proceedings of the 47th Annual Meeting of the American Fertility Society, S91.

5) Katayama, K.P., Stehlik, E. \& Jeyendran, R.S. (1989) In vitro fertilization outcome: Glass-wool-filtered sperm vs swim-up sperm. Fertil. Steril., 52, 670-672.

6) Kofinas, G.D. \& Zavos, P.M. (1992a) Short-term cryostorage technique for human spermatozoa: Its possible application in an artificial insemination program. Infertility, 15, 44-54.

7) Kofinas, G.D. \& Zavos, P.M. (1992b) Selection of viable spermatozoa via sperm filtration following $24 \mathrm{hr}$ cryostorage at $5^{\circ} \mathrm{C}$ in test-yolk buffer. Mol. Androl., 4, 113119.

8) McClure, D.R., Nules, L. \& Tom, R. (1989) Semen manipulations improved sperm recovery and function with a two-layer percoll gradient. Fertil. Steril., 51, 874-877.

9) Rogers, B.J. (1985) The sperm penetration assay: Its usefulness reevaluated. Fertil. Steril., 43, 821-840.

10) Rogers, B.J., Wamil, B. \& Zavos, P.M. (1991) Comparison of the fertilizing potential of human spermatozoa processed by swim-up or Sephadex filtration columns. Proceedings of the 7th World Congress on IVF and Assisted Procreations, 318.

11) Sofikitis, N., Miyagawa, I. \& Zavos, P.M. (1992a) The SpermPrep ${ }^{\text {TM }}$ filtration column selectively entraps single-stranded DNA spermatozoa. Jpn. J. Fertil. Steril., 37, 384-387.

12) Sofikitis, N., Miyagawa, I., Zavos, P.M., Inaga, S., Iino, A., Toda, T., Harada, T., Mio, Y. \& Terakawa, N. (1992b) Acrosin profiles of human spermatozoa recovered from the new SpermPrep ${ }^{\mathrm{TM}}$ II filtration column. Tohoku J. Exp. Med., 166, 451-452.

13) Tanphaichitr, N., Millete, C.F., Agulnick, A. \& Fitzgerald, L.M. (1988) Eggpenetration ability and structural properties of human sperm prepared by Percollgradient centrifugation. Gam. Res., 20, 67-73.

14) World Health Organization (1987) WHO Laboratory Manual for the Examination of Human Semen and Semen-Cervical Mucus Interaction, 2nd edition, Cambridge, The Press Syndicate of the University of Cambridge, p. 62.

15) Zavos, P.M. (1985) Characteristics of human ejaculates collected via masturbation and a new Silastic seminal fluid collection device (SCD). Fertil. Steril., 43, 491-492.

16) Zavos, P.M. (1990) Principles of cryopreservation of human spermatozoa: State-ofthe-art. Infertility, 13, 239-246.

17) Zavos, P.M. \& Goodpasture, J.C. (1989) Clinical improvements of specific seminal deficiencies via intercourse with a seminal collection device versus masturbation. Fertil. Steril., 51, 190-193.

18) Zavos, P.M. \& Centola, G.M. (1990) Qualitative and quantitative improvements in human spermatozoa recovered via the swim-up and a new semen filtration method. Infertility, 13, 25-34.

19) Zavos, P.M. \& Centola, G.M. (1992) Methods of semen preparation for intrauterine insemination and subsequent pregnancy rates. Tohoku J. Exp. Med., 168, 583-590.

20) Zavos, P.M., Goodpasture, J.C., Zaneveld, L.J.D. \& Cohen, M.R. (1980) Motility and enzyme activity of human spermatozoa stored for 24 hours at $+5^{\circ} \mathrm{C}$ and $-196^{\circ} \mathrm{C}$. Fertil. Steril., 34, 607-609.

21) Zavos, P.M., Wilson, E.A. \& Cohen, M.R. (1984) Total functional sperm fraction measurements in males of known fertility and infertility. Fertil. Steril. 41, 29S. 
(abstract)

22) Zavos, P.M., Sofikitis, N., Miyagawa, I. \& Toda, I. (1992) Selection and preparation of human spermatozoa for artificial insemination using the new and improved SpermPrep ${ }^{\mathrm{TM}}$ II filtration method. Jpn. J. Fertil. Steril., 37, 388-393. 